\title{
EXTERNAL ForCE For DEFORMABLE MODELS IN MEDiCAL IMAgE SEgMENTATION: A SURVEY
}

\author{
Basavaraj Amarapur ${ }^{1}$ and P. K. Kulkarni ${ }^{2}$ \\ ${ }^{1}$ Department of Electrical \& Electronics Engineering, Poojya Doddappa Appa College of \\ Engineering, Gulbarga, Karnataka, India \\ bamarapur@yahoo.com \\ 2 Department of Electrical \&Electronics Engineering, Poojya Doddappa Appa College of \\ Engineering, Gulbarga, Karnataka, India \\ drpkkulkarni@yahoo.com
}

\begin{abstract}
Many image segmentation techniques are available in the literature. Among the available techniques, parametric deformable models play an important role in many medical imaging applications. These models have been proved to be effective in segmenting an anatomic structure using constraints derived from the image data along with a prior knowledge about the location, size and shape of these structures. These models also support highly intuitive interaction mechanisms, which helps medical practitioners to bring their expertise to bear on the model-based image interpretation task. In this paper, a review of nineteen different external forces for the parametric deformable model applied to the medical image segmentation is presented. The main purpose of survey is to identify and discuss each category with its principle, mathematical model, advantages, disadvantages and applications to medical image analysis.
\end{abstract}

\section{KEYWORDS}

Deformable models, Gradient vector flow, Segmentation, External force, Medical images.

\section{INTRODUCTION}

Nowadays in clinical practice, images are commonly used to provide a means for proper diagnosis and as an aid in planning a therapy. The main goal of medical image analysis includes interpretation, analysis \& visualization as a means for surgical planning \& simulation, postoperative progression of the disease, and intra operative navigation. There are different image modalities such as X-ray, Computed Tomography (CT), Single Photon Emission Computed Tomography (SPECT), Position Emission Tomography (PET), Ultrasound (US) and Magnetic Resonance Imaging (MRI), which are applied to acquire different tissue properties. Each modality is based on different imaging physics, and thus reveals different aspects of the anatomy or physiology.

The segmentation of these modalities is a challenging task because of image quality and speckle noise present in the image. Due to presence of noise, it is very difficult to extract an accurate boundary of region of interest using segmentation algorithm. At the same time, limited information on true boundaries makes it nearly impossible to evaluate the segmentation algorithms. In such difficult circumstances, it is expected that segmentation error may occur. The result of such errors will be the imprecise definition of regions in images and pixel will be wrongly assigned to a different region or object affecting its statistical distribution. In order to overcome these difficulties, a methodology that can encapsulate local statistics in a global framework might prove to be a better alternative in this respect. Extensive research in this area has resulted in many deformable models $[1,2]$. These are distinguished mainly by the type of DOI : $10.5121 /$ sipij.2011.2208 
curve representation used and the choice of the image energy term. The popular curve representation schemes in the deformable model literature are

1. Point based deformable models, where the curve is an ordered collection of discrete point $[3$, 5].

2. Parametric deformable models, where the curve is described continuously in a parametric form using basis function on such as B-splines [6-9], Fourier exponentials [10-11] etc.

3. Geometric deformable models, where the planar curve is represented by Level set of an appropriate 2-D surface [12-16].

If the interested structures have open boundaries or thin structure or algorithms need real time operations, then parametric models are preferred. These models play an important role in many medical imaging applications $[5,17,18,19,24,29]$ using automated delineation of anatomical structures and other region of interest. These models support highly intuitive interaction mechanisms, which allow medical practitioners to use their expertise on the model-based image interpretation task.

In this paper, reviews of nineteen different external forces for the parametric deformable models as applied to the medical image segmentations are presented. The main purpose of survey is to identify and discuss each category with their principle ideas, mathematical models, advantages, disadvantages and applications to medical image analysis as given in the following section.

\section{PARAMETRIC DEFORMABLE MODEL}

The parametric deformable model or active contour proposed by Kass et al [04] is an elastic contour that deforms under the guidance of attributed geometric and image properties. This phenomenon of deformation, as guided by physical laws, is defined in terms of an energy minimization framework. By definition, the minimization of the total energy over the entire shape is given by the equation as follows:

$$
E=\int_{0}^{1} \frac{1}{2}\left(\alpha\left|X^{\prime}(s)\right|^{2}+\beta\left|X^{\prime \prime}(s)\right|^{2}\right)+E_{e x t}(X(s)) d s
$$

The first part of the integral is related to the deformable model's internal energy and imposes restrictions to its movement by controlling the elasticity \& stiffness parameters, which are weighed by $\alpha$ and $\beta$, respectively. The second part stands for the external energy, and is responsible for driving the deformable model towards important features in the image. The external energy for a grey level image I $(\mathrm{x}, \mathrm{y})$ can be written as

$$
E_{e x t}(x, y)=-\left|\nabla\left(G_{\sigma}(x, y) * I(x, y)\right)\right|^{2}
$$

Where $\mathrm{G}_{\sigma}(\mathrm{x}, \mathrm{y})$ is a Gaussian filter applied to the image in order to improve the image's edge map, and also to perform some noise reduction. This makes regions closer to edges of the gradient term that yields higher values, and in consequence, stronger forces. A local minimization of equation (1) can be found by resolving the Euler-Lagrange equation on $\mathrm{x}$.

$$
\alpha X^{\prime \prime}(s)-\beta X^{\prime \prime \prime \prime}(s)-\nabla E_{e x t}=0
$$

The above equation can be viewed as a force balancing equation, where the term $\alpha X^{\prime \prime}(s)$ $\beta X^{\prime \prime \prime \prime}(s)$ corresponds to the internal force. $-\nabla E_{\text {ext }}$ corresponds to external potential force that pulls the snake towards the desired image edges.

By treating $X(s)$ as a time dependent function and its solution is obtained by using $t=0$. The corresponding equation is given as follows. 


$$
X_{t}(s, t)=\alpha X^{\prime \prime}(s)-\beta X^{\prime \prime \prime \prime}(s)-\nabla E_{e x t}=0
$$

When the deformable model reaches steady state, the term $X_{t}(s, t)$ disappears and a solution is found from equation (3). The model can be solved numerically using finite difference techniques.

In spite of the model's consistency and simplicity, there are some performance problems associated with the model such as initialisation, stopping criteria and impossibility of converging to concave boundaries etc. In order to overcome the above problems, the external force component of equation (3) can be modified to improve the deformable model capture range and to force towards the image edges. As a result, researchers have proposed various external force models. The following sub sections describe the most important models, their advantages and disadvantages.

\subsection{Pressure force or Balloon model}

The initialisation problem can be improved by adding a constant normal force component to the external force term [19] and is given as follows:

$$
F_{e x t}=k_{1} \bar{n}(s)-k \frac{\nabla E_{e x t}}{\left\|E_{e x t}\right\|}
$$

Due to this force, deformable model may inflate or deflate depending on the initialisation of deformable curve and magnitude of the balloon force. In equation (5), $\bar{n}(s)$ is the normal unit vector with magnitude $k_{1}$ and $k$ is the external force weight. When image gradient force balances with the inflating or deflating force, then the deformable contour fits on the object boundary and stops the desirable contour moment.

The main drawback of this model is that it may become strong enough to ignore the image gradient force in week object edges.

\subsection{Adaptive Pressure model}

Chaoquan Chen et al [20] proposed an Adaptive pressure force by modifying the balloon force as an additional force. This force is able to derive the deformable contour towards the desired boundary in both directions and is given as follows:

$$
E_{\text {ext }}=-k F_{\text {ext }}+k_{p} \bar{n}(s)
$$

Where $\bar{n}(s)$ is the normal unit vector with magnitude $\mathrm{k}_{\mathrm{p}}$, and $\mathrm{k}$ is external force weight. The adaptive pressure will drive the contour towards the desired boundary from both directions within the catching range specified by $r$ in the following equation:

$$
\left(x_{n}-x\right)^{2}+k\left(x_{n}-x\right)^{2} \leq r^{2}
$$

Where $\left(x_{n}, y_{n}\right)$ is a pixel along the normal and $\mathrm{k}$ gives the normal direction of the contour at the pixel $(\mathrm{x}, \mathrm{y})$. In the traditional balloon model, the pressure force may inflate or deflate depending on the initialisation of deformable curve. But this model is able to drive the contour towards the desired boundary in both directions. 


\subsection{Distance Potential Force Model}

The distance potential force [21] defined based on the Euclidian distance can be applied to increase the capture range of deformable model. The external force of deformable model is replaced by normalized distance potential force as follows:

$$
E_{\text {ext }}(x, y)=-\frac{k \nabla d(x, y)}{\|\nabla d(x, y)\|}
$$

Where $\mathrm{k}$ is a constant and $\mathrm{d}$ is the distance between a point $(\mathrm{x}, \mathrm{y}) \&$ the nearest edge points in the binary boundary map. Distance potential force based deformable model have high capture range but it cannot extract concave objects and sharp corners correctly.

\subsection{Gradient vector flow (GVF) model}

To solve the problems of limited capture range and poor convergence of traditional model, Chenyang and Prince [22] proposed a new external force for deformable model by replacing $-\nabla E_{e x t}$ with GVF field $V(s)=[u(s), v(s)]$ that minimizes the following object function:

$$
E=\iint \mu\left(u_{x}^{2}+v_{x}^{2}+u_{y}^{2}+v_{y}^{2}\right)+|\nabla f|^{2}(V-\nabla f)^{2} d x d y
$$

Where $u_{x}, u_{y}, v_{x}, v_{y}$, are the spatial derivatives of the field, $\mu$ is the blending parameter, $f$ is an edge map of the input image I and $|\nabla f|$ is the gradient of the edge map defined as the negative external force which is computed by data-driven and regulation terms. The data- driven term dominates this function in the object boundaries (i.e. $|\nabla f|$ is large), while the regulation term dictates the function in areas where the information is constant (i.e. $|\nabla f|$ tends to zero).

The GVF is calculated by solving the following Euler equations with the help of calculus of variations. The normalized GVF is calculated and used as the static external force for deformable model as given bellow:

$$
\mu \nabla^{2} u-\left(u-f_{x}\right)\left(f_{x}^{2}+f_{y}^{2}\right)=0 \quad(10), \mu \nabla^{2} v-\left(v-f_{y}\right)\left(f_{x}^{2}+f_{y}^{2}\right)=0
$$

In equation (10) and (11), $\nabla^{2}$ is a Laplacian operator, $f_{x}$ and $f_{y}$ are partial derivative of $f$. The GVF is computed as a diffusion of the gradient vectors of grey level or binary edge map derived from the image. The resultant field has a large capture range and tends to force deformable contours into boundary concavities. It still has difficulties such as noise sensitivity, unable to deform into long $\&$ thin boundaries, and the ambiguous relationship between the capture range \& parameters.

\subsection{Generalized Gradient Vector Flow (GGVF) model}

GVF has difficulty of forcing a deformable model into long and thin boundary of region of interest. This difficulty could be caused by excessive smoothing of the field near the boundaries, governed by the co-efficient $\mu$ in equation (9). Xu et al [23] introduced a special varying weight functions $g$ (.) and $h$ (.) in place of $\mu$ and $|\nabla f|^{2}$ in equation (9). The resultant energy equation and weighting functions for GGVF are as given bellow:

$$
E_{G G V F}(u, v)=\iint g(|\nabla f|)\left(u_{x}^{2}+v_{x}^{2}+u_{y}^{2}+v_{y}^{2}\right)+(1-g(|\nabla f|))\left((V-\nabla f)^{2}\right) d x d y
$$




$$
g(|\nabla f|)=\exp \left(-\left(\frac{|\nabla f|}{k}\right)\right) \quad(13), h(|\nabla f|)=1-g(|\nabla f|)
$$

The $\mathrm{k}$ is a user-defined parameter controlling the degree of smoothness of the deformable model force field. The GGVF vectors are obtained using following equations.

$$
g \nabla^{2} u-(1-g)\left(u-f_{x}\right)=0 \quad(15), g \nabla^{2} v-(1-g)\left(v-f_{y}\right)=0
$$

The main advantage of this model is to support convergence to very thin boundary concavities, while simultaneously eliminating extraneous edge points. This model performs excellently on noisy as well as real medical images.

\subsection{Merging deformable model}

Nilanjan ray et al [24] proposed the external guiding force for the deformable model. This model was designed such that two non-intersecting as well as growing deformable contours never cross each other and never leave any space between contours unless an object exists in between. Additionally, they require that the growing deformable models stop at the object boundary. In this regard, the external force field was computed on the image domain by solving GGVF partial differential equations with the Dirichiet boundary conditions based on the initial deformable model.

The bounding conditions of the Dirichlet type are as given bellow:

$$
\begin{gathered}
V(x, y)=0, \quad \text { for }(x, y) \in D \\
V(x, y)=n(x, y) \in \partial D, \text { for }(x, y) \in \partial D
\end{gathered}
$$

The $\mathrm{n}(\mathrm{x}, \mathrm{y})$ is the unit outward normal at $(\mathrm{x}, \mathrm{y})$ on the image domain boundary $\partial D$. In essence, this is treated as the initial deformable curve with the sources emitting unit normal vectors on the image domain. Solving the equation (12) along with bounding condition as given in equation (17), results in the required guiding force for the deformable models with merging capability. Thus, it diffuses the gradients of the edge map and the outward normal vectors defined on the initial deformable model.

\subsection{Discrete cosine transform based GVF model}

A new gradient vector flow deformable model presented by Jinshan et al [25] based on the discrete cosine transform (DCT) for object boundary detection. In order to develop this model, for each pixel in the spatial domain, the two dimensional DCT was computed in its local neighbourhood. A contrast measure derived from the local DCT co-efficient is used to compute the gradient vector flow field, which is employed as the external force to drive the deformable model to the object edges. The local contrast of the image at the given pixel location $(k, l)$ is defined as

$P(x, y)=\frac{\sum_{t=1}^{2(2 n+1)-1} W_{t} E_{t}}{d_{00}}$

Where $E_{t}=\frac{\sum_{v+u=t}\left|d_{u, v}\right|}{N}$ is the average amplitude over a spectral band and defined as the coefficient set with similar frequency as given bellow: 


$$
\begin{array}{lrl}
\mathrm{N}=t+1 & t<2 n-1 \\
N=2(2 n+1)-t & t \geq 2 n+1
\end{array}
$$

Where $\mathrm{W}_{\mathrm{t}}$ denotes the weights used to select the DCT co-efficient. Using P in equation (18) or its threshold version, the new energy function was obtained by replacing $P$ by the edge map $f$ in equation (12), and is given as follows:

$$
E_{G V F-D C T}(u, v)=\iint g\left(\left|\nabla_{P}\right|\right)\left(u_{x}^{2}+v_{x}^{2}+u_{y}^{2}+v_{y}^{2}\right)+\left(1-g\left(\left|\nabla_{P}\right|\right)\right)\left(V-\nabla_{P}\right)^{2} d x d y
$$

The DCT based GVF are obtained by replacing P by $f$ in equations (15) and (16). The advantage of this model lies in its robustness to multiplicative noise.

\subsection{Edge Preserving Gradient Vector Flow (EPGVF)}

In this model, an Edge Preserving Gradient Vector Flow (EPGVF) was employed as an external force field [26]. The orientation of the field is obtained by the following equation:

$$
p=\left[\frac{-I_{y}}{\sqrt{I_{x}^{2}+I_{y}^{2}}}, \frac{I_{y}}{\sqrt{I_{x}^{2}+I_{y}^{2}}}\right]^{T}
$$

Then the EPGVF defined as the vector field $\mathrm{V}=(\mathrm{u}, \mathrm{v})$ that minimizes the following functional $\mathrm{E}$ :

$$
E(V)=\iint\left[g(x, y)\left(|\nabla u|^{2}+|\nabla v|^{2}\right)+h(x, y)\left(\mu\left|J_{v} p\right|^{2}+|V-\nabla f|^{2}\right)\right] d x d y
$$

Where $\mathrm{g}(\mathrm{x}, \mathrm{y})$ and $\mathrm{h}(\mathrm{x}, \mathrm{y})$ are the weighting functions; $\mu$ is a positive parameter, and $\mathrm{J}_{\mathrm{v}}$ is the Jacobean matrix of the vector field V. The first term of equation (22) is called smoothing term and second term is called edge-preventing term. By choosing appropriate weighting functions $\mathrm{g}$ and $\mathrm{h}$, one can achieve the goal of extending the capture range and at the same time preserving object boundaries.

This method overcomes the drawbacks of boundary smearing associated with GVF and GGVF. Moreover the segmented EPGVF field provides a novel and effective mechanism for automatic initialisation and splitting of deformable models. Also it prevents the deformable model from passing over weak boundary.

\subsection{GVF and adaptive balloon model}

Suhuai luo et al [27] developed an extension of the dynamic GVF model based on combined use of two external forces such as adaptive balloon and dynamic GVF force. The adaptive balloon force is used to increase the capture range and convergence speed of GVF model. The dynamic GVF force is introduced to provide an efficient evolution-stop mechanism. The new external force is given as follows:

$$
\begin{array}{rlr}
V d y n & =V & \text { if } \mathrm{C} \Theta>\mathrm{T} \Theta \\
& =\frac{-\alpha X^{\prime \prime}(s)+\beta X^{\prime \prime \prime}(s)-\lambda f_{p}}{\gamma} & \text { Otherwise }
\end{array}
$$

Where $V_{d y n}$ is a dynamic gradient vector flow force, $f_{p}$ is the balloon force, Ce defines the consistency angle proportional to the angle between the GVF vectors and cut-off angle is represented by Te. The new dynamic GVF force is same as conventional GVF force if the deformable contour point moves towards the contour. But when the deformable model point tries 
to cross over an edge, the dynamic GVF force will stop the point from moving. The threshold Te will decide when this evolution-stop mechanism will be triggered. This model is robust to the variation in initial position and efficient in preventing the deformable model from breaking through correct contour and locking to other feature points.

\subsection{Directional Gradient Vector Flow (DGVF)}

In order to make the GVF deformable model more stable and to converge, a new model developed $[28,29]$ by incorporating direction information in the gradient vector flow model. This can be obtained by replacing $\mathrm{f}$ by $\mathrm{F}$ in the equation (12), results in the direction gradient vector flow of the image by minimizing the equation (24).

$$
E_{D G V F}(u, v)=\iint g(|\nabla F|)\left(u_{x}^{2}+v_{x}^{2}+u_{y}^{2}+v_{y}^{2}\right)+(1-g(|\nabla F|))\left((V-\nabla F)^{2}\right) d x d y
$$

Where $\quad F=D_{n}(I)$ if $D_{n} I>0$

$$
=0 \quad \text { otherwise }
$$

$\mathrm{D}_{\mathrm{n}}(\mathrm{I})$ is an edge map function that incorporates gradient direction information and is given as:

$$
D_{n}(I)=\nabla I \bullet n
$$

Where $\mathrm{I}$ is the original image, $\nabla I$ is the gradient of $\mathrm{I}$, and $\mathrm{n}$ is a two-dimensional vector that represents the direction of the edge as specified by the user. The directional GVF vectors are obtained by solving the equations:

$$
g \nabla^{2} u-(1-g)\left(u-F_{x}\right)=0 \quad(27), \quad g \nabla^{2} v-(1-g)\left(v-F_{y}\right)=0
$$

\subsection{Dynamic Directional Gradient Vector Flow (DDGVF) model}

GVF deformable model defines the external energy in terms of gradient magnitude of the image and is a conventional step edge detector. As the magnitude operator discards the signs of gradient, the deformable model is unable to distinguish between positive and negative step edge. To deal with this problem, Jierong Chens et al [30] proposed a new external force model called as dynamic directional gradient vector flow (DDGVF) to distinguish between positive boundary and negative boundary which is given as:

$$
E_{\text {ext }}=\left\lfloor E_{x}, E_{y}\right\rfloor
$$

For grey-level images, a boundary is defined to be positive, if there are positive step edges along its outward normal. Contrarily, a boundary is defined to be negative step edges along its outward normal, and $\cos \Theta \& \sin \Theta$ are normal vector components in the " $\mathrm{x}$ " and " $\mathrm{y}$ " directions respectively, which are given as follows:

$$
\begin{aligned}
& E_{x}=u^{+} * \max \{\cos (\theta), 0\}-u^{-} * \min \{\cos (\theta), 0\} \\
& E_{y}=v^{+} * \max \{\sin (\theta), 0\}-v^{-} * \min \{\sin (\theta), 0\} \\
& \text { Where } u^{+}=\mu \nabla^{2} u^{+}-\left(u^{+}-d f_{x}^{+}\right)\left(d f_{x}^{+}\right)^{2}(32), u^{-}=\mu \nabla^{2} u^{-}-\left(u^{-}-d f_{x}^{-}\right)\left(d f_{x}^{-}\right)^{2} \\
& v^{+}=\mu \nabla^{2} v^{+}-\left(v^{+}-d f_{y}^{+}\right)\left(d f_{y}^{+}\right)^{2} \quad \text { (34), } v^{-}=\mu \nabla^{2} v^{-}-\left(v^{-}-d f_{y}^{-}\right)\left(d f_{y}^{-}\right)^{2}
\end{aligned}
$$

Where $f_{x}^{+}, f_{x}^{-}, f_{y}^{+}$and $f_{y}^{-}$are the gradients of positive step edges in $\mathrm{x},-\mathrm{x}, \mathrm{y}$ and -y directions and they are the directional edge map. The $\mathrm{E}_{\mathrm{x}}$ and $\mathrm{E}_{\mathrm{y}}$ are the horizontal and vertical external forces. 


\subsection{Vector Field Convolution (VFC) model}

Bing $\mathrm{Li}$ et al [31] developed a new external force for deformable model. This force was calculated by convolving the user defined vector field kernel $k(x, y)$ with the edge map $f(x, y)$ derived from the image $\mathrm{I}(\mathrm{x}, \mathrm{y})$ and is given as follows:

$$
f_{v f c}(x, y)=f(x, y) * k(x, y) \quad(36), f_{v f c}(x, y)=\left[f(x, y) * u_{k}(x, y), f(x, y) * v_{k}(x, y)\right]
$$

The vector field kernel is given by the following equation:

$$
k(x, y)=m(x, y) n(x, y)
$$

Where $\mathrm{n}(\mathrm{x}, \mathrm{y})$ is the unit vector pointing to the kernel origin $(0,0)$ and $\mathrm{m}(\mathrm{x}, \mathrm{y})$ is the magnitude of the vector at $(\mathrm{x}, \mathrm{y})$, which are given as:

$$
n(x, y)=\left[\frac{-x}{r}, \frac{-y}{r}\right] \quad(39), m_{1}(x, y)=(r+\varepsilon)^{-\gamma}(40), m_{2}(x, y)=\exp \left(\frac{-r^{2}}{\varsigma^{2}}\right)
$$

Where $r=\sqrt{\left(x^{2}+y^{2}\right)}$ the distance from origin, $\gamma$ and $\zeta$ are positive parameters to control the decrease, $\varepsilon$ is a small positive constant to prevent division by zero at the origin. The advantages of this method include superior noise robustness, reduced computational cost, and the flexibility of tailoring the force field. The disadvantage of this method is that the weak edge might be over whelmed by the strong edges along with the noise.

\subsection{Improved Compound Vector Field Model}

In this model, an improved deformable model algorithm for image segmentation based on compound vector field was proposed [32]. The image is processed by the generalized fuzzy theory to get a better edge map. The proposed model achieves the better effect on image segmentation by replacing GVF field with compound vector field as follows:

$$
F_{\text {ext }}=\rho(\nabla f) V(x, y)+\psi(\nabla f) \nabla f
$$

$\rho(\nabla f)=\exp (-(\nabla f) / k)$

$$
\text { (43), } \psi(\nabla f)=1-\exp (-(\nabla f) / k)
$$

$\rho(\nabla f)$ is the dynamic constraint weight, it ensures that the GVF will play the major role as the force field appears in the consistent region of image, $\psi(\nabla f)$ ensures that the gradient force controls the motion of curve as it starts convergence near the target edges. The main advantage of this model is brilliant capacity of not only capturing the image feature in a wider range but also dealing with the concave regions.

\subsection{Boundary Vector Field (BVF) model}

Boundary vector field [33] extends the capture range further to the entire image based on interpolation. A threshold is applied to generate a binary boundary map of the input image. Then, four potential functions $\Psi \mathrm{x}, \Psi \mathrm{y}, \Psi \mathrm{xy}$, and $\Psi_{\mathrm{yx}}$ are computed using line-by-line interpolations in the horizontal, vertical, and two diagonal directions. The Bounded vector fields are defined based on the gradients of the following potential functions:

$$
\phi_{1}=\left\lfloor\nabla \psi_{x}, \nabla \psi_{y}\right\rfloor \quad(45), \phi_{2}=\left[\frac{1}{\sqrt{2}}\left(\nabla \psi_{x y}+\nabla \psi_{y x}\right), \frac{1}{\sqrt{2}}\left(\nabla \psi_{x y}-\nabla \psi_{y x}\right)\right]
$$

The external force is given as follows: 


$$
E_{e x t}(x, y)=\phi(x, y)
$$

The advantages of this model are faster alternative with enhance capture range and concave object extraction capability. The disadvantage of this model is inability to extract acute concavities.

\subsection{Deformable super ellipses model}

A parametric shape modelling using deformable super ellipse for segmentation of medical image was proposed [34]. The external force calculated by incorporating of optimal shape information in the process of segmentation is given as follows:

$E_{e x t}(x, y)=-\lambda_{1}\left|\nabla\left(G_{\sigma}(x, y)\right)\right|^{2}-\lambda_{2}\left|\left(f(p)^{*}-f(p)\right)\right|$

Where $\left(f(p)^{*}-f(p)\right)=\sum_{i}\left(\left(x_{i}^{*}-x_{i}\right)^{2}+\left(y_{i}^{*}-y_{i}\right)^{2}\right)$

$\left(x_{i}^{*}, y^{*}{ }_{i}\right)$ is a mean position of initial points on the optimal super ellipse and $\left(x_{i}, y_{i}\right)$ is a mean of the new positions of the initial points computed from the deformable model algorithm. The advantages of this model are less sensitivity to initial contour placement and more robustness even in the presence of large boundary gaps.

\subsection{Simulated Static Electric Field (SSEF) model}

Dan Yuan et al [35] proposed external force for deformable model. The external force is created by simulated static electric charges. The force in this field was computed by the Coulomb K. Law and is given by the following equation with respect to the direction:

$$
F=\sum_{i} \frac{\overline{\nabla f_{i}}}{r^{2}+\varepsilon}
$$

Where $\overline{\nabla f_{i}}$ is the $\mathrm{i}_{\mathrm{th}}$ high gradient point on gradient map of the image, $\mathrm{r}$ is the distance from the $\mathrm{i}_{\text {th }}$ charge and $\varepsilon$ is a constant with a small value. Each point in the two dimensional space has two directions: vertical \& horizontal, and sum of each force from every charge in both directions. Finally, it was obtained by the summation of $F$ at each point. The advantages of this model are the generated forces pointing to deeper \& narrower concavities and less sensitive to the noise.

\subsection{Virtual electric field}

A new external force [36] was proposed based on electric field, which is made by virtual electric charges that are defined as edges or intensities in an image. The electric field at (x,y) is created by the electric charge placed at origin and is given by

$$
E_{1}=\left(\frac{q_{x}}{4 \pi \varepsilon r^{3}}, \frac{q_{y}}{4 \pi \varepsilon r^{3}}\right)
$$

Where $r=\sqrt{\left(x^{2}+y^{2}\right)}$ and $\varepsilon$ is the primary constant, if $\mathrm{n}$ electric charges are placed, then the total electric field is computed by the vector sum of each electric field. The virtual charge $q$ of equation (51) is defined in terms of intensities or the edges of the image and is directly given by

$$
q_{\text {image }}(x, y)=-I(x, y) \quad(52), q_{\text {edge }}(x, y)=-|\nabla I(x, y)|^{2}
$$

Final external force for the deformable model is given by: 


$$
F_{\text {ext }}=\frac{E}{|E|}
$$

This external force has same properties as that of GVF and it takes the less computation time.

\subsection{Self-Affine model}

M Saadatmand-Tarzjan et al [37] developed an external force for parametric deformable model. The external force was computed by integrating the wavelet transform and self-affine mapping system as given by

$$
f_{S A S}(p)=f_{S G}(p)+\sum_{m=1}^{n} \eta^{-m} f^{m}\left(p / \eta^{m}\right)
$$

The $f_{S A S}(p)$ indicates the self-affine force at component $p$ of the image, $\eta$ is the scaling coefficient of the wavelet decomposition and $\mathrm{f}_{\mathrm{SG}}$ determines a sub-set of Gaussian force as given by:

$$
f_{S G}(p)=\frac{f_{G}(p)}{\max \left\|f_{G}(p)\right\|} \quad \frac{\left\|f_{G}(p)\right\|}{\max \left\|f_{G}(p)\right\|}>\theta
$$

$$
0 \quad \text { otherwise }
$$

The $\mathrm{f}_{\mathrm{G}}$ is the potential Gaussian force defined as :

$$
f_{G}(p)=-\nabla\left(\left\|\nabla \mid G_{\sigma S A S} * I(p)\right\|^{2}\right)
$$

The remarkable advantages include robustness against noise $\&$ intensity in homogeneity, wide capture range, progressing into boundary concavities, and short processing time.

\subsection{Fluid Vector Flow (FVF) model}

A new external energy function developed by Tao wang et al [38] to enhance the capture range, which is defined as

$$
\begin{aligned}
E_{F V F}(x, y) & =\chi\left(f_{x}+\partial \cos \phi, f_{y}+\partial \sin \phi\right) & & \text { When } M_{B B}(x, y)=0 \\
& =0 & & \text { otherwise }
\end{aligned}
$$

Where $\chi$ is a normalized operator, $\delta= \pm 1$ (controls the inward or outward direction when the contour is outside or inside), $\left(f_{x,} f_{y}\right)=\chi(\nabla I(x, y))$ and

$$
\begin{array}{lc}
\phi=\arctan \left(\frac{y-y_{c}}{x-x_{c}}\right) & x \neq x_{c} \\
\phi=\pi / 2 & \text { When } x=x_{c} \text { and } y>y_{c} \\
\phi=\pi / 4 & \text { When } x=x_{c} \text { and } y<y_{c}
\end{array}
$$

Where $\phi \in[0,2 \pi], M_{B}(x, y)=\left|\nabla\left(-G_{\sigma}(x, y) * I(x, y)\right)\right|$ and $\left(x_{c}, y_{c}\right)=\left(\sum_{i=0}^{P-1} x_{i} / P, \sum_{i=0}^{P-1} y_{i} / P\right)$. The normalized boundary map is computed by the following equation: 


$$
M_{N B}(x, y)=\frac{M_{B}(x, y)-\min \left(M_{B}(x, y)\right)}{\max \left(M_{B}(x, y)\right)-\min \left(M_{B}(x, y)\right)}
$$

Then binary boundary map is calculated using threshold value i.e., $T \in|0,1|$. This is given by equation as follows:

$$
\begin{aligned}
M_{B B}(x, y) & =1, M_{N B}(x, y)>T \\
& =0 \text { otherwise }
\end{aligned}
$$

The external energy $\mathrm{E}_{\mathrm{FVF}}$ has two components namely: directional and gradient components. When the deformable model is far away from the object, the directional force dominates and attracts the deformable model towards the object and is computed based on a polar transformation. When the contour is close to the object, the gradient force fits the deformable model to the object and is computed in a similar manner as the traditional and GVF deformable model. The capture range of FVF extends to the whole image because the vector flow energy defined in equation (61) spreads around the entire image I (x,y). Even if the initial deformable model is far from the object, the deformable model can still evolve toward the object. This feature makes FVF more effective than either the traditional or the GVF deformable model. Although the capture range of BVF can extend to the entire image, the performance of FVF is more efficient because interpolation is avoided. Furthermore, the BVF interpolation is executed in only four directions, whereas FVF is direction invariant $(\varphi \in[0,2 \pi])$.

\section{APPLICATIONS TO MEDICAL IMAGE ANALYSIS USING PARAMETRIC DEFORMABLE MODELS}

The Parametric deformable models are applied to different image modalities such as Magnetic resonance image $[05,17,18,24,27,29,32,33,37,38,39,40]$, Ultrasound [20, 31, 42, 43, 44], Computed tomography $[29,34,45]$ and $\mathrm{X}$-ray [46]. These are used to segment, visualize, track and quantify, a variety of anatomic structures ranging from the macroscopic to the microscopic such as brain, heart, lung, liver and calculi, etc. In present section deals with the application of parametric deformable models applied to medical image interpretation.

The segmentation of the interior contour of the brain from MR images of the head by the combined use of an Artificial Neural Network (ANN) and Traditional Deformable Model (TDM) [40] was carried out. The ANN is trained to produce a binary classification of each pixel as either a boundary or a non-boundary point. Subsequently, the resulting binary (edge-point) image forms the external energy function for the TDM, which is used to link the region of interest boundary points into a continuous closed contour. However, the initial classification achieved by the neural network in this work is relatively poor and requires a complex model-based deformable model to extract the final boundary. Early results for the classification stage, using data from a single subject and a restricted number of slices, were reported by Middleton et al [48], and have shown the better segmentation of the lung boundaries in a given MR image of the torso. Unfortunately, however, generalization to other slices and subjects was very much poor. In order to overcome this difficulty, several modifications and improvements were made and further, the lungs were successfully segmented [18] from the vast majority of available images using a balloon model [19]. Nilanjan et al [24] proposed a method to capture the total lung cavity space in proton of MR image using merging parametric deformable model. It was compared with the balloon force model and observed that the balloon model leaks through the low contrast edges; where as this deformable model survives both the weak as well as strong edges.

The segmentation of the endocardium of left ventricle of human heart was carried out using GVF model $[05,17]$. The better effect on image segmentation was achieved by replacing the GVF with 
the GGVF [23], Mean Shift Flow Field (MSEF) [39], compound vector field [32] and boundary vector field [33]. The corresponding results are shown in fig. 1(a), 1(b), 1(c), 1(d) and 1(e) respectively. From the result, it is found that BVF model increases the capture range, improved in concave object extraction capability in noise image, reduces the computational requirements as compared to other methods.

The segmentation of MR image of knee \& blood cells were carried out using distance, balloon and GVF deformable models [49]. From the segmented results, it was observed that the GVF model requires less number of deformation steps as compared to distance and balloon models. Suhuai Luo et al [27] proposed a new method to segment a tumour in brain MR images. Method

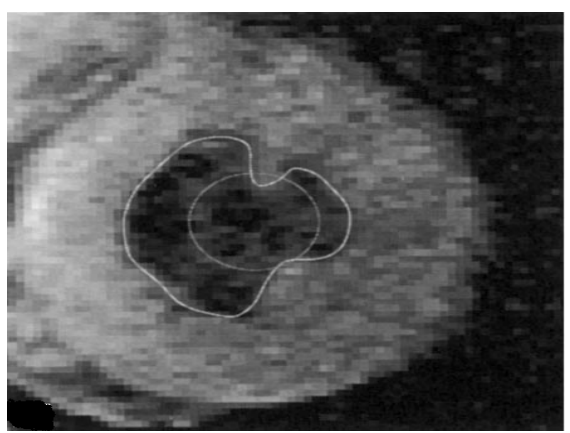

$1(\mathrm{a})$

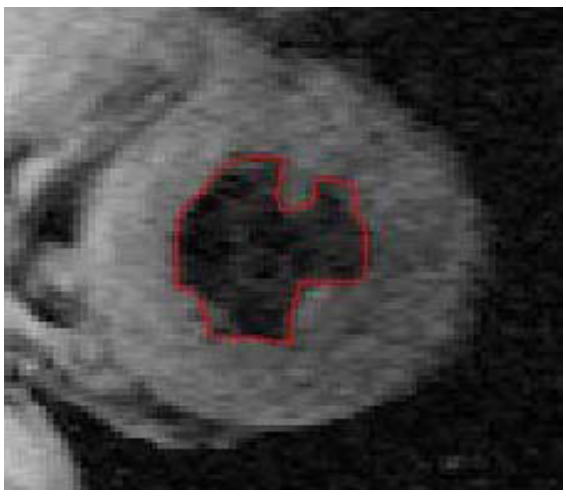

$1(\mathrm{c})$

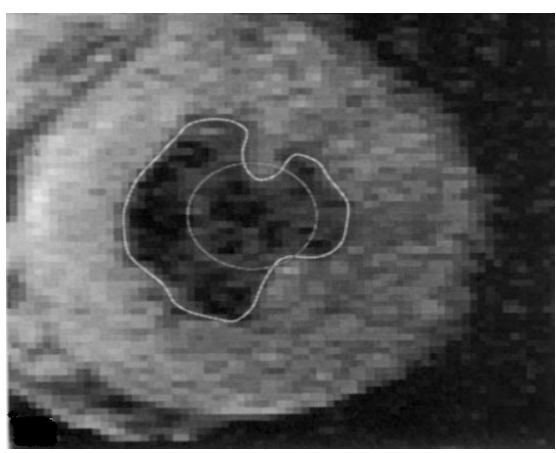

$1(\mathrm{~b})$

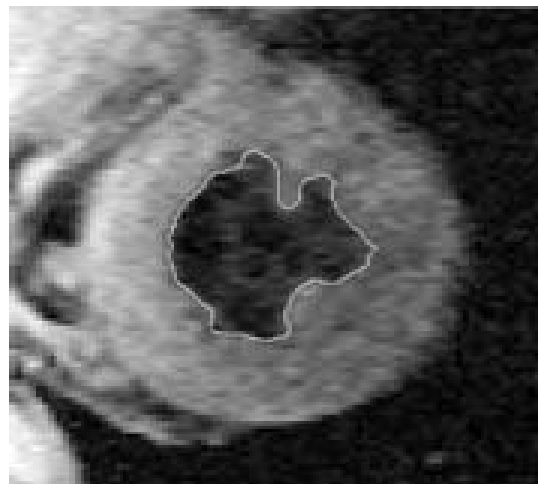

$1(\mathrm{~d})$

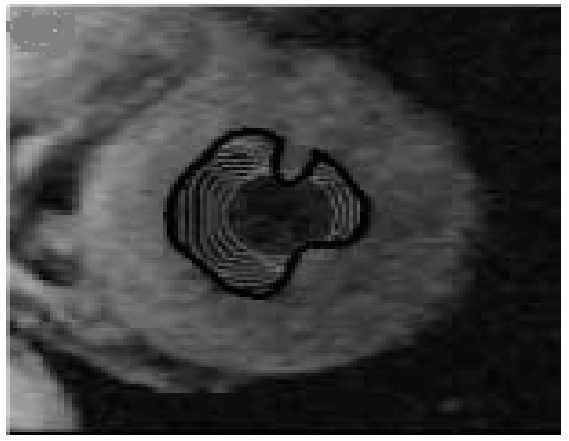

$1(\mathrm{e})$

Fig 1: Segmented result of endocardium of MRI using (a) GVF (b) GGVF (c) MSEF-GVF (d) compound vector flow (e) BVF 
is based on combining of balloon force and GVF models. An adaptive balloon force was introduced to increase GVF deformable model's capture range and speed up evolution. Then the dynamic GVF force is used to provide an efficient evolution-stop mechanism. It shows that the proposed method is immune to the variation in initial position and effective in preventing the deformable model from breaking through correct contour and locking to other feature points. The segmentation of femoral candles \& tibia from a coronal and four cardiac MR images of human knee and heart respectively were carried out using self-affine deformable model. The model was developed by integration of the wavelet transform and self-affine mapping system to effectively compute the external force of parametric deformable model [37]. From the result, they demonstrated that the better performance as compared to GVF and GGVF for medical images. Tao Wang et al. [38] segmented the pediatric head and brain tumour from MR images using fluid vector flow model.

The segmentation of calculi was carried out from various positions of US images such as gall bladder, renal and urinary bladder acquired from different patients. The original image of urinary bladder calculi with initial contour is as shown in fig 2(a) and the segmented results of traditional and GVF models are shown in fig 2(b) and 2(c) respectively.

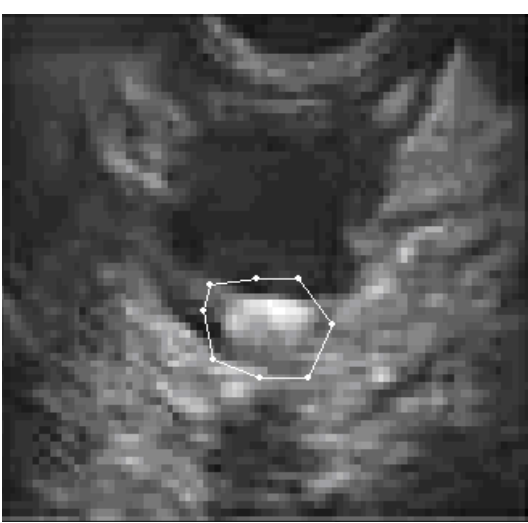

$2(a)$

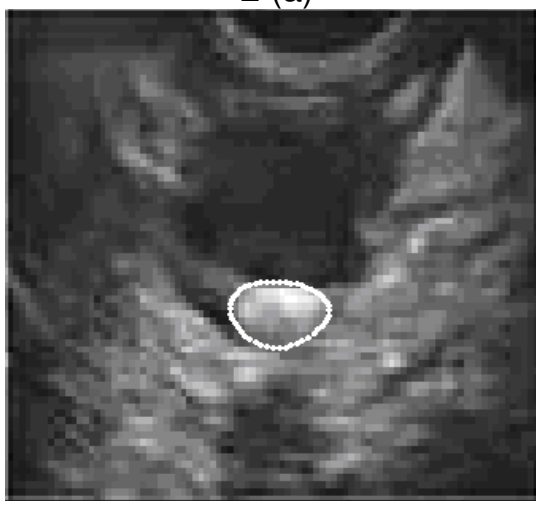

2 (c)

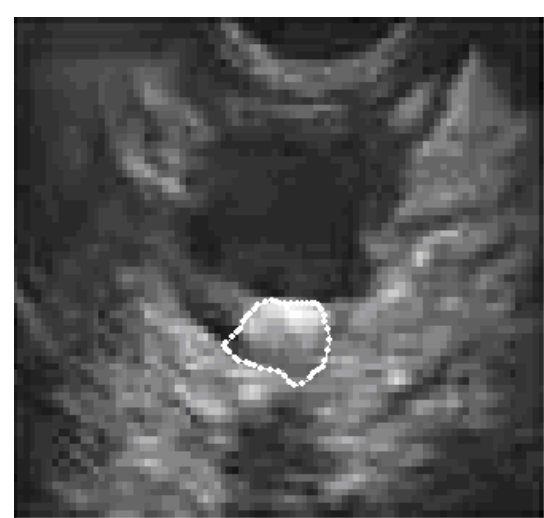

$2(b)$

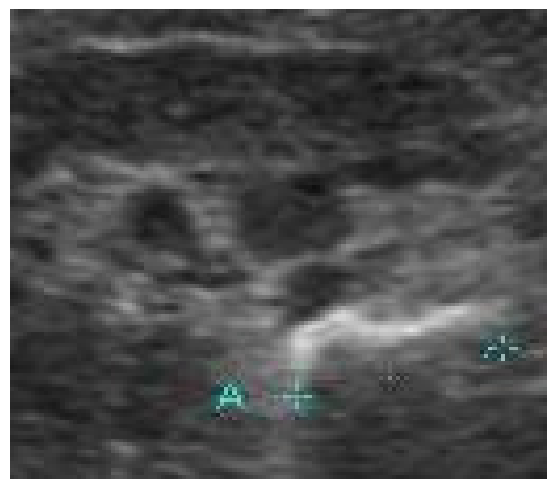

2 (d)

Fig 2(a): Original image of urinary bladder calculi with initial contour, (b) Processed with traditional deformable model, (c) Processed with GVF deformable model, (d) Original image of right renal calculi

From the result, it is concluded that the accuracy and robustness of the shape descriptions obtained by GVF model on ultrasound images out performs TDM [43, 44]. In case of more speckle noise present in US image of calculi of right renal as shown in fig 2(d), the GVF deformable model segmentation algorithm fails to segment the right portion calculi image as 
shown in fig 2(f). In order to overcome this difficulty a hybrid model was proposed [42] by combining speckle reducing an isotropic diffusion model applied along with GVF deformable model. The developed model was applied to ultrasound image of renal calculi in two steps. In the first step, noise was reduced by an isotropic diffusion model on log-compressed image by adjusting number of iterations, time step and selecting a rectangular window of uniform intensity area using trial and error method as shown in fig $2(\mathrm{~g})$. In second step, the GVF deformable model was applied for segmentation of speckle noise reduced image as shown in fig 2(h). From the evaluation, it was concluded that the accuracy and robustness of the shape descriptions obtained by hybrid model on ultrasound images out performs GVF deformable model for noisy images. It was also observed that the edges are more prominent and visible as compared to the original image

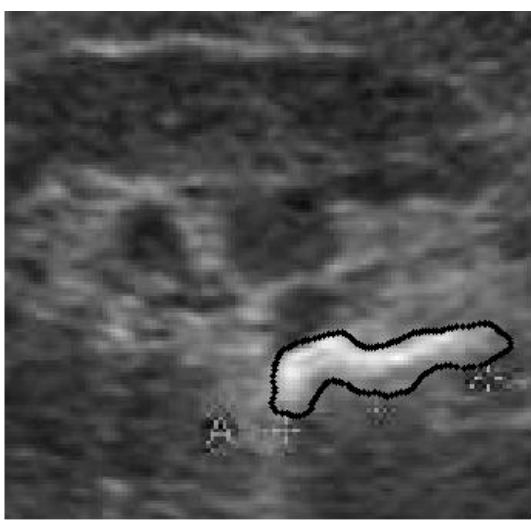

$2(\mathrm{e})$

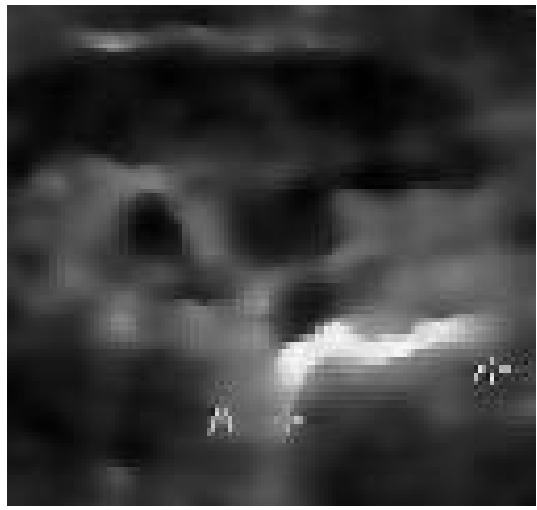

$2(\mathrm{~g})$

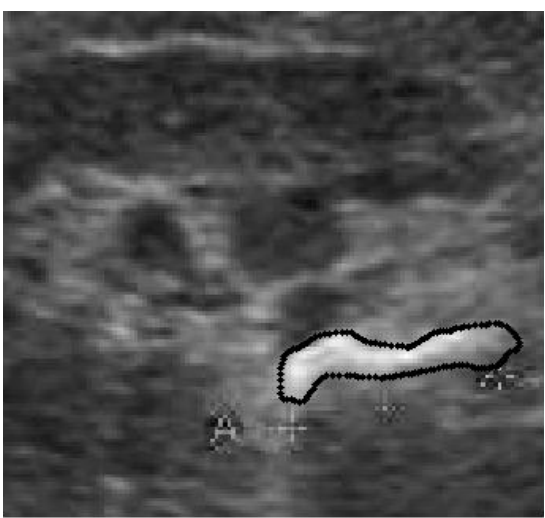

2 (f)

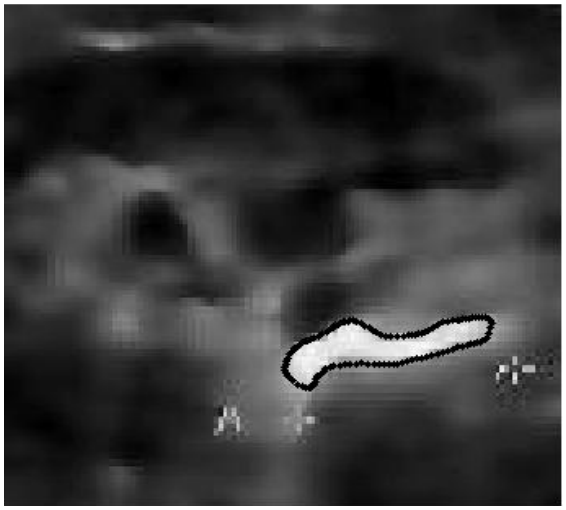

$2(\mathrm{~h})$

Fig 2(e) Processed with traditional deformable model and (f) processed with GVF deformable model, (g) processed with speckle an isotropic reduced diffusion filter, (h) Processed with hybrid model.

The segmentation of tumour on breast ultrasound images [50] was carried out using balloon and GVF models. The segmented results were compared with the manual segmentation in terms of area of tumour. The original image of cyst, results of segmented image using balloon and GVF are as shown in fig 3(a), fig 3(b) and fig 3(c) respectively. From the results, it was observed that the balloon model is more suitable for this application as compared to GVF model.

The GVF, DGVF and DDGVF deformable models were applied to ultrasound images of echocardium of the left ventricle of human heart and comparison between them was carried out by Jierong cheng et al [30]. The original image is as shown in fig 4(a) and results of segmentation 
of using GVF, DGVF and DDGVF models are as shown in fig. 4(b), 4(c) and 4(d) respectively. The GVF deformable model fails to detect the real boundaries, at the regions where the potential force propagating from the desired boundary is smaller than that of local noise. Being attracted by not only the positive edges, the GVF deformable model tends to move to the exterior of the endocardium. This problem was overcome by using DGVF deformable model. This is able to discern positive boundary from negative boundary and works better than the GVF deformable model. However, due to the deficiency of edge map, the DGVF deformable model is not able to reach sharp convex in the contour. This difficulty was solved by DDGVF deformable model. DGVF is only attracted to the positive boundaries and is capable of distinguishing the desired edges from the false edges. Thus DDGVF deformable model was shown to be superior to GVF and DGVF snake models for the segmentation of ultrasound images.

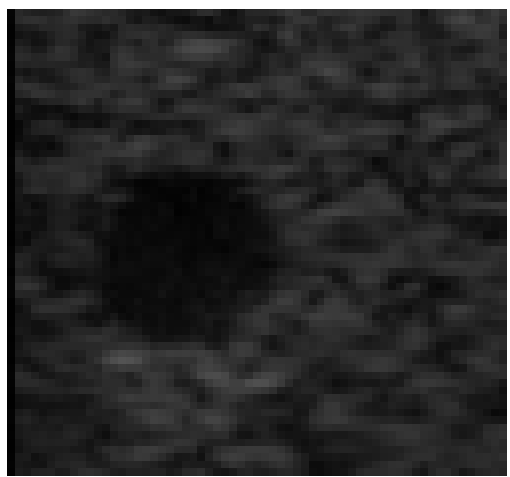

3(a)

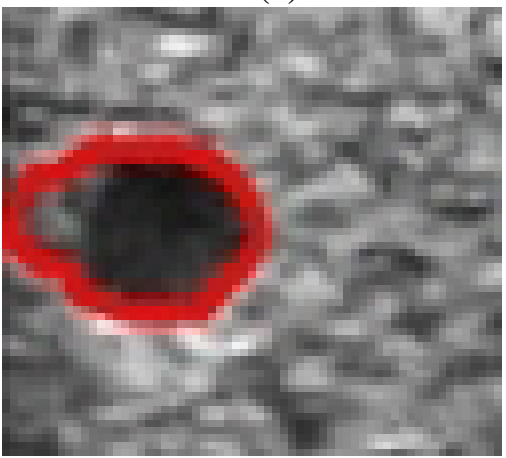

$3(\mathrm{c})$

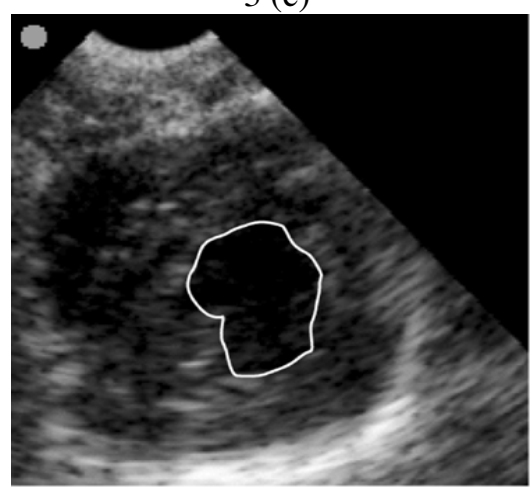

4(b)

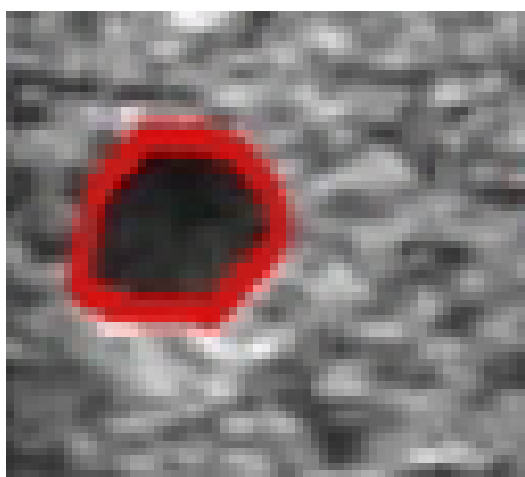

3(b)

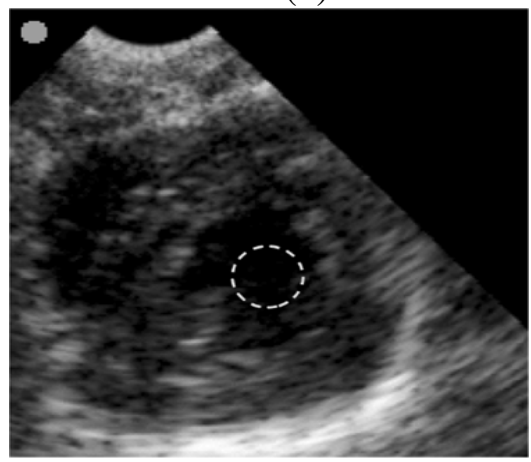

4 (a)

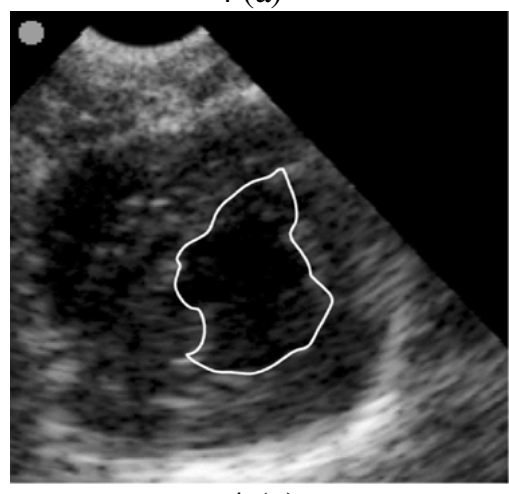

4 (c)

3(a): Original ROI Cyst Mass, (b) Segmented image using Balloon and (c) Using GVF deformable model. 4 (a): Original echocardiograph image of ultrasound with the initial deformable contour (b) segmented result using GVF (c) segmented result-using DGVF. 


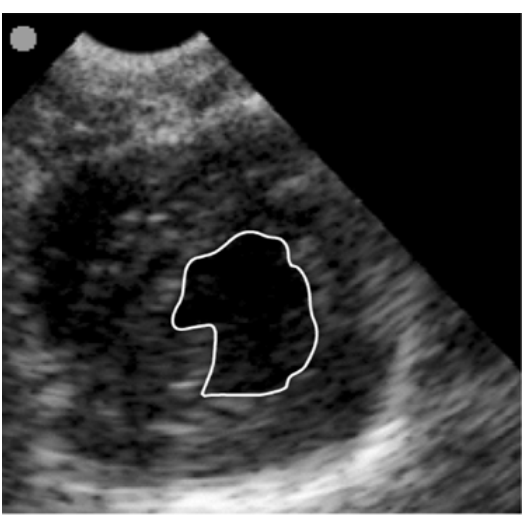

$4(d)$

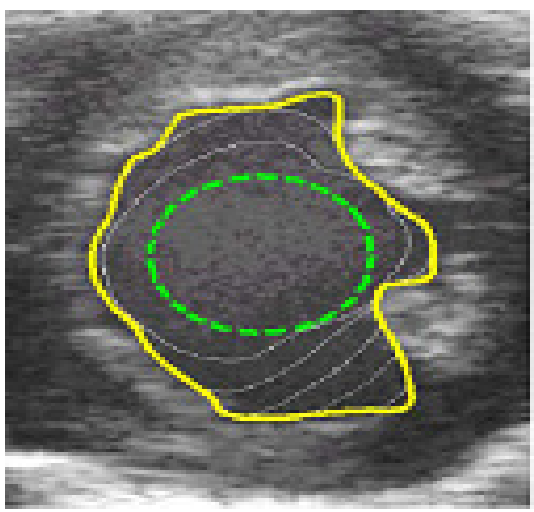

5 (b)

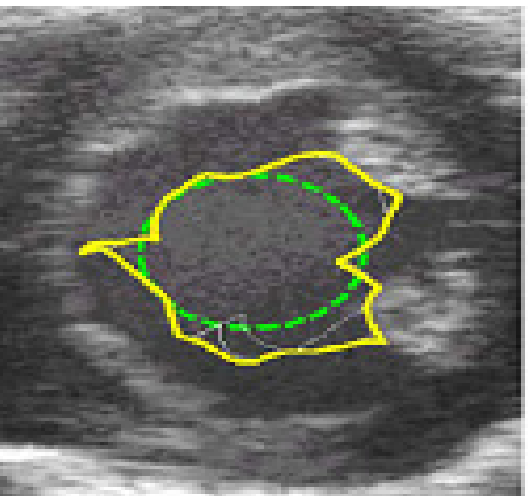

5 (a)

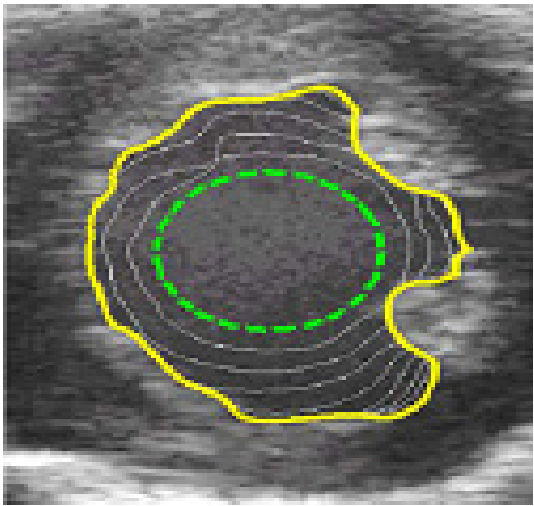

$5(\mathrm{c})$

Fig 4(d): Segmented image using DDGVF deformable models. Fig 5: Ultrasound image of a mouse heart with initial snake and results using (a) GVF, (b) DDGVF, and (c) VFC field deformable model.

Ruey-Feng Chang et al [50] developed a technique to segment the tumour in US image of breast. In this technique, first the speckle noise is reduced using an isotropic diffusion filter. Then the stick detection is adopted for enhancing the edge. Finally, the GVF deformable model is used to obtain the tumour contour and the evaluation for the breast tumour excision, which enables the operator to inspect the performance of the surgery. The segmentation of carotid arteries in US images using adaptive pressure was carried out [20] and is able to drive the contour towards the desired boundary in both the directions instead of inflate or deflate pressure. The mouse heart from US images was segmented using VFC deformable model [31]. The results were compared with GVF and DDGVF models as shown in fig 5. From the fig. 5(a) it is observed that the GVF snake becomes stuck in the interior and does not converge to boundary. This problem occurs due to GVF deformable model tending to move in the vertical direction since there is little horizontal force component in the central area. It was also noted that the GVF deformable model converges, if the initialisation is closer to the boundary as shown in fig 5(b). In contrast, the VFC deformable model converges to the boundary concavities on the lower right precisely from the same initialisation at the centre as shown in fig 5(c). From the above results Bing li et al [31] concluded that the VFC model is computationally less expensive and most robust to noise \& initialisation.

An interactive method based on the adaptive threshold segmentation of tumour in CT image was presented [51]. The method is based on the adaptive threshold segmentation to accurately extract a general initial contour. Then the GVF deformable model with this initial contour was used to 
segment the desired object. The experimental results show that the tumour can be segmented with only little user interaction. Segmentation of the right trapezius muscle from the CT image using deformable super ellipse model [34] was carried out as shown in fig 6. The CT image of right trapezius and the corresponding segmented image are as shown in fig 6(a) and 6(b) respectively. From the result, it was observed that the algorithm is less sensitive to initial contour placement and more robust even in the presence of large boundary gaps. Carl Philips et al [45] proposed a hybrid approach for liver segmentation of CT images. The proposed approach that combines texture based features, principal component analysis and gradient vector flow to produce a deformable model based segmentation algorithm invariant to the grey level overlap presented by soft tissues.
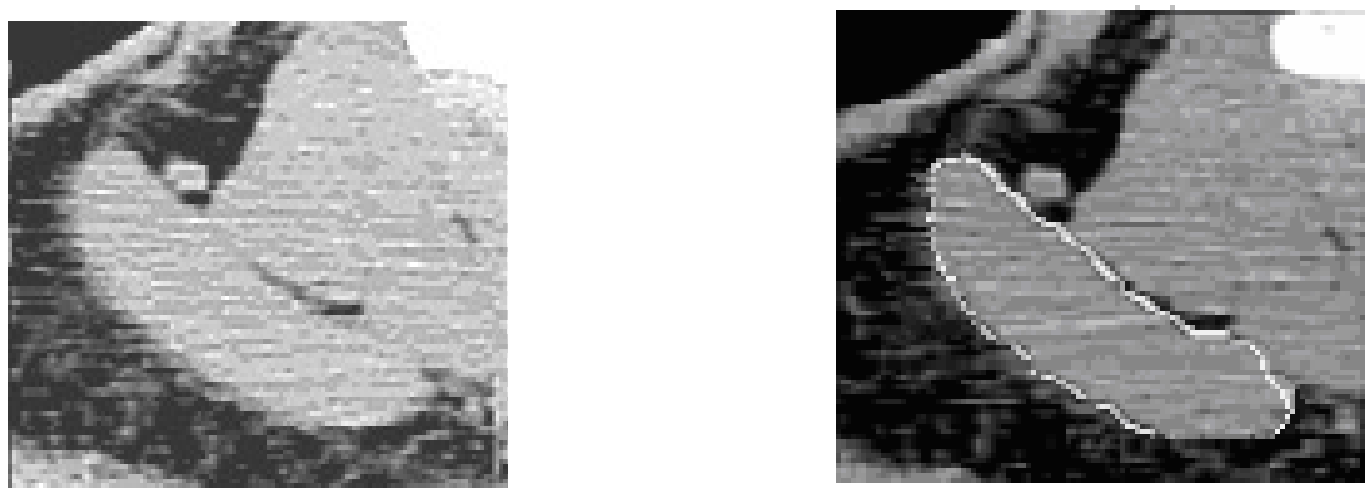

Fig 6: (a) CT image of the right trapezius muscle, and (b) result of segmentation

Sridharan K. et al [46] developed an approach of DGVF deformable model to detect the double edges in X-ray images of lumbar vertebral using spine radiographs. In this method, they combined open contoured DGVF deformable models with appropriately defined pressure forces to achieve accurate detection of vertebral double edges in the presence of noise and neighborhood of adjacent vertebrae.

\section{CONCLUSIONS}

The important role of medical imaging in the diagnosis and treatment of diseases has opened various challenging problems in order to accurately segment the anatomic structures from medical images. Parametric deformable models offer an attractive approach to tackle such problems, by providing compact \& analytical representations of object shape incorporating anatomic knowledge and interactive capability. The continued development and refinement of these models still remains an important area of research. This paper presents a review of nineteen external force models for parametric deformable model and their versatility and shortcomings. It also presents the applications of models to segmentation of widely used medical image modalities such as CT, MRI, ultrasound, and X-ray. From the review, it is concluded that even though there are still many challenges to be overcome, it is worth to understand both the problems and the features provided by the various methods that a successful solution could be devised. One important point to be noted here is, these methods are not mutually exclusive. The better segmentation results can be obtained by integrating the evolution framework with more information from image or object. For example, the deformable contour method considering both edge and region features can obtain better results than those based on only edge information. Segmentation methods will be particularly suitable in areas such as image-guided surgery - in which visualization of the anatomy is critical component, surgery planning and post operated result analysis. The present paper provides a better insight to both the biomedical image segmentation problems and the powerful features of the parametric deformable model. 


\section{REFERENCES}

[1] A. K. Jain, Y. Zhong and M.P.D. Jolly,"Deformable template models: A review", Signal processing, vol 76, pp 109-129, 1998.

[2] T. McInerney and Terzopoulos, "Deformable models in medical image analysis: A survey", Medical Image Analysis, vol 1, pp 91-108, 1998.

[3] Mathews Jacob, Thierry Blu and Michael User, "Efficient energies and algorithms for parametric snakes", IEEE transactions, Image Processing, Jan 7, 2004.

[4] M. Kass, A. Witkin and D. Terzopoulos, (1987), "Snakes: Active Contour Models", International Journal Computer Vision, Vol. 1, pp 321-331.

[5] Chenyang Xu and Jerry L. Prince, (1998), "Generalized gradient vector flow external force for active contours, Elsevier- Signal Processing, vol 71, pp 131-139.

[6] J. Gao, A. Kosaka, and A. Kak, “A deformable model for human organ extraction”, ICIP, Chicago, pp 323-327, 1998.

[7] S. Menet, P. Saint- mark and G. Medioni, "B- Snakes: Implementation and application to stereo", Image understanding work shop, pp 720-726, 1990.

[8] M. Gebhard, J. mattes and R. Eiles," An active contour model for segmentation based on cubic Bsplines and gradient vector flow", MICCAI, 2001.

[9] M. A. Figueiredo and J. M. N. Letao, "Unsupervised contour representation and estimation using bsplines and minimum description length criteria", IEEE transaction on Image Processing, vol 9, No 6, pp 1075-1087, 2000.

[10] P.brigger, J. Hoeg and M. User, “ B-splines snakes: A flexible tool for parametric contour detection”, IEEE Transaction on Image Processing, vol 9, No 9, pp 1484-1496, 2000.

[11]L. H. Staib and J. S. Duncan, "Boundary fitting with parametrically deformable model", Machine Intelligence, vol 14, No 11, pp 1061-1075, 1992.

[12] A. Chakkraborthy, L. H. staib, and J. S. Duncan, "Deformable boundary finding in medical images by integrating gradient and region information", IEEE Transaction on medical imaging”, vol 15, no 6, pp 859-870.

[13] V. Caselles, R. Kimmel and G. Sapiro, "Geodesic active contours", Fifth International conference on computer vision, pp 694-699, 1995

[14] R. Mallabadi, J A. Sethian and B. C. Vemuri, "Shape modeling with front propagation: A level set approach, IEEE Transactions on Pattern Annual, Machine Intelligence”, Vol 17, No. 2, pp 158-175, 1995.

[15]J. A. Sethian, "Level set methods and fast marching methods: Evolving interfaces in computational geometry, fluid mechanics, computer vision and material science", Cambridge, U. K. Cambridge University, 1999

[16] G. Sapiro, "Geometric partial Differential equation and image analysis", Cambridge university press, 2001.

[17] Chenyang. Xu and J. L. Prince, "Snakes, shapes, and Gradient Vector Flow", IEEE Transactions on Image Processing, vol. 7, No. 3, pp 359-369, 1998.

[18] Ian Middleton and Robert I. Damper, "Segmentation of magnetic resonance images using a combination of neural networks and active contour models", Medical Engineering and physics vol 26, pp 71-86, 2004.

[19] Laurent. D. Cohen, “An Active Contour models and balloons”, CVGIP: Image Understanding, Vol. 53, No. 2, pp211-218, 1991 
Signal \& Image Processing : An International Journal (SIPIJ) Vol.2, No.2, June 2011

[20] Chaoquan Chen, Tamie L, Jason J, Steven H, Richard B, Bill H, Paul A, William J and Peter R, "Segmentation of arterial geometry from ultrasound images using balloon models", IEEE International conference proceedings, pp1319-1322., 2004

[21] Laurent. D. Cohen and Isaac. Cohen, (1993), "Finite-Element methods for active contour models and balloons for 2-D and 3-D Images", IEEE Transactions on Pattern Analysis and Machine Intelligence, Vol. 15,No. 11, pp 1131-1147.

[22] Chenyang Xu and Jerry L. Prince, "Generalized gradient vector flow external force for active contours, Elsevier- Signal Processing, vol 71, pp 131-139, 1998.

[23] Chenyang. Xu and J. L. Prince, "Snakes, shapes, and Gradient Vector Flow”, IEEE Transactions on Image Processing, vol. 7, No. 3, pp 359-369, 1998.

[24] Nilanjan. Ray, Scott T. Talissa. Altes, Eduard E., D. Lange, and James, R. Brookeman, (2003), "Merging Parametric Active Contours Within Homogeneous Image Regions for MRI Based Lung Segmentation", IEEE Transactions on Medical Imaging, Vol. 22, No. 2, pp189-199.

[25] Jinshan Tang and Scott T.," A DCT Based gradient vector flow snake for object boundary detection”, IEEE International conference, pp 157-161, 2004.

[26] Chunming. Li, Jundong. Liu and Martin. Fox, " Segmentation of Edge Preserving Gradient vector Flow: An Approach toward Automatically Initializing and Splitting of Snakes", Proceedings of IEEE conference On Computer Vision and Pattern Recognition, pp1063-1069, 2005.

[27] S. Luo and Li. R, "A new deformable model using dynamic vector flow and adaptive balloon forces", APRS Workshop on Digital image computing, Australia, pp 9-14, 2003.

[28] Jinshan. Tang, S. Millington, Scott A., J. Crandall and S. Hurwitz, "Surface extraction and Thickness measurement of the Auricular cartilage from MR image using directional gradient vector flow snakes", IEEE Transactions on Biomedical Engineering, Vol. 53, No. 5,pp 65-80, 2006.

[29] Park H. Wm and Schoepflin T. Kim, "Active contour model with gradient directional information: directional snake", IEEE Transactions Circuit System Video Technology, Vol 11, pp 252-256, 2001.

[30] Jierong. Cheng and Say Wei, Foo, ” Dynamic Directional Gradient Vector Flow for Snakes”, IEEE Transactions on Image Processing, vol. 15, No. 6, pp 1563-1571, 2006.

[31] Bing. Li, and Scott T. Action, (2007), "Active contour external force using vector field convolution for image segmentation”, IEEE Transactions on Image processing, vol. 16, No. 8, pp 2096-2105.

[32] Yang Gao and Wufan Chen, "Improved compound vector field based active contours model", IEEE International Conference,pp 1-5, 2009.

[33] K.W. Sum, Paul Y. S. and Cheung, "Boundary vector field for parametric active contours, Pattern Recognition", Vol 40, pp 1635-1645., 2007.

[34] Min Xiaoping, Wang Boliang, Huang Shaohui and Dai Peishan, "Parametric shape modeling using deformable super ellipse for segmentation of medical image”, IEEE, pp 2673-2676, 2008.

[35]Dan Yaun and Siwei Lu, "Simulated static electric field (SSEF) snake for deformable models", International Conference IEEE, 2002.

[36] Hyun Keun Park and Myung Jin Chung, "External force of snake: Virtual electric field, Electronic Letters", Vol 138, No 24, pp 1500-1502, 2002.

[37] M. Saadatmand-Tarzjan and H. Ghassemian, "Self-Affine snake: A new parametric active contour", IEEE International Conference on Signal Processing and Communications,pp 24-27, 2007.

[38] Tao Wang, Irene Cheng, and Anup Basu, " Fluid Vector Flow and Applications in Brain Tumor Segmentation”, IEEE Transactions on Biomedical Engineering, vol. 56, no. 3, pp 781-89, 2009.

[39]A. Guocheng, Chen Jianjun and Wu Zhenyang, "A fast external force model for snake based image segmentation", ICSP proceedings, pp 1128-1131, 2008 
Signal \& Image Processing : An International Journal (SIPIJ) Vol.2, No.2, June 2011

[40] Chiou G. I. and Hwang J. N., " A neural network-based stochastic active model (NNS-SNAKE) for contour of distinct features", IEEE Transactions Images Processing: vol 4, No 10, pp 1407-1416, 1995.

[41] Lie He, Zhigang Pens, Bryan Everdins, Xun wang, Chain Y Han, Kenneth L, and William G, “A comparative study of deformable contour methods on medical image segmentation", Image and Vision Computers, 26, pp 141-163, 2008.

[42] Basavaraj Amarapur and P.K. Kulkarni, "A New approach for feature extraction from ultrasound images using hybrid model", International Journal of Emerging Technologies and Applications in Engineering, Technology and Science (IJ-ETA-ETS), Vol 3, No 1, pp506-511, 2010.

[43] Basavaraj Amarapur and P.K. Kulkarni, "Computer-Aided Diagnosis Applied to Ultrasound Image of Calculi using Gradient Vector Flow model”, International Journal of Tomography and Statistics, Vol 12, No 12,pp 113-128, 2009.

[44] Basavaraj Amarapur and P.K. Kulkarni, (2010), "Computer-Aided Diagnosis Applied to Ultrasound Image of Calculi using hybrid model”, International Journal of Computer Applications in Engineering, Technology and Science, Vol 3, Issue 2, pp 172-178, 2010.

[45] Carl Philps, Reem mokhtar, Ruchaneewan Susomboon, Daniela Raicu and Jacob Furst, (2007) "Segmentation of soft tissue using texture features and gradient snakes", IEEE International conference proceedings.

[46] Sridharan Kamalakannan, Arunkumar G, Hameed Sari-Sarff, Rodney long and Sameer Anatani, "Double edge detection of radiographic lumbar vertebrae images using pressurized open DGVF snakes", IEEE Transactions on Biomedical Engineering, Vol 57, No 6, pp 1325-1334, 2010

[47]Jenn-Lung Su, Hsuan-Yu, Chun-Yi, Hsiao-Yen Chang and Yeum-Chang, "The selection of characteristic for lesions image in X-ray mammography and breast sonography for computer aided diagnosis system development”, IFMBE Proceedings, vol 14, part 15, pp 2607-26112007.

[48] Middleton I and Damper R I., "Segmentation of magnetic resonance of the thorax by back propagation", Proceedings of IEEE International conference on neural network, Vol 15, pp 2490-2494, 1995.

[49] Abdul Kadir, Wan E. Zarina, W. A. Rahman, Arsmah I. and Rozi Mahmud, "Comparison of balloon snake and GVF snake in segmentating masses from breast ultrasound images", IEEE international conference proceedings, pp 505-509, 2010.

[50] Ruey Feng-chang, Wen-Jie Wu, Chin-Chi T Seng, Dar-Ren Chen and Woo Kyung Moon, “3-D snake for US margin evaluation for malignant breast tumor excision using mammotone", IEEE Transactions on Information Technology in Biomedicine, Vol. 7, No. 3. pp 197-201, 2003.

[51] Wu Bingrong, Xie Mei, Li Guo and Go Jingjing," Medical image segmentation based on GVF snake model", Proceeding of ICICTA, 2009.

[52] Ahmed Jendoubi, Jianchao Zens, Mohamed F and Chouikha, (2009), "Segmentation of Prostate ultrasound image using an improved snakes model”, ICSP Proceeding, 04, pp2568-2570.

\section{Authors}

Basavaraj Amarapur completed B.E. Electrical Engineering from H. K. E. Society's Engineering college Gulbarga in the year 1985 and Masters in computer science \& Engineering from University of Roorkee in the year 1991.Currently he is working as Assistant Professor in the department of Electrical and Electronics Engineering, Poojya Doddappa Appa college of Engineering Gulbarga, Karnataka, India. $\mathrm{He}$ is submitted his Ph. D. Thesis to VTU Belgaum. His research area of interest is Biomedical Image Processing and Pattern Recognition.

P. K. Kulkarni completed B. E. Electrical Engineering from H.K.E Society's Engineering College in the year 1983 and Masters in Instrumentation from I. I. T. Kharagpur in the year 1989 and Ph. D in the area of Biomedical Instrumentation from University of Roorkee in the year 1998. Presently he is working as Professor and Head of Electrical and Electronics Dept., Poojya Doddappa Appa College of Engineering Gulbarga, Karnataka, India. He is currently working in the area of Biomedical Signal \&Image Processing. 